\title{
Lab Animal Management syllabus, part 1
}

\begin{abstract}
Along with their responsibilities for training investigators and animal care providers, many trainers also teach lengthy didactic courses that lead to certification or form part of a school curriculum. I am one of those. For almost 10 years, I have taught a course called Laboratory Animal Management and Rules and Regulations, which consists of 30 hours instructional time (3 hours per week for 10 weeks) along with a hands-on lab. It is part of an American Veterinary Medical Association (AVMA)-accredited program to train vet techs about animal research. As a result of this course, many students, who thought only about dog and cat clinical work, 'discover' lab animal science and obtain jobs in research. These positions allow the techs to work with a wide variety of species, learn about science and contribute to important discoveries. The management topics are, of course, also useful in other animal enterprises like veterinary clinics, zoos and shelters.
\end{abstract}

I want to share my syllabus so that others might pick and choose from its 'fruits'. In this column, I include the first four weeks; the rest will come next month. The full syllabus is 10 pages long, so some details are not included here.

The course objectives are as follows: (i) learn elements of managing a lab animal facility meant primarily for biomedical research; (ii) become aware of regulations and performance standards in lab animal management; (iii) learn the components necessary for a suitable lab animal program to become accredited by the Association for Assessment and Accreditation of Laboratory Animal Care International; (iv) complete exercises related to actual tasks in the lab animal facility; (v) personally consider the privileged use of animals in research and other disciplines; (vi) know that research and teaching with animals is more than biomedical (i.e., environment and behavior science); and (vii) appreciate the welfare and ethics associated with lab animal usage.

Primary resources include $L A T G$ Training Manual and AALAS in a Flash flashcards (American Association for Laboratory Animal Science (AALAS), Memphis, TN, 2006), Management of Laboratory Animal Care and Use Programs (CRC Press, Boca Raton, FL, 2001; reading assignments in the table below refer to this text), Managing the Laboratory Animal Facility (CRC Press, Boca Raton, FL, 2009), internet sites and vendor materials.

Students are assigned to write a hypothetical protocol based upon their own interests, which forms the backbone for other assignments. For example, a student may choose to study treatments for Alzheimer's disease in rats, then could write a protocol using cranial electrode implants, prepare a budget of the materials, consider specific veterinary care and learn which regulations apply to the species.

\section{Presentation topic and material highlights}

Philosophy of the course

Various uses of animals (food, testing, service, companions); proponents (advocates) and opponents of lab animal research; concept of a model; devising a protocol (hypothesis, research plans, statistics, variables, justification of use of an animal, scientific merit); how lab animal research supports veterinary and human health

Principal investigators (PIs) and their responsibilities; kinds of animal models, their care and applicable regulations

Managing care (the veterinary component)

Co-presented with a former vet tech student working in research. Vet care; role of vet technicians and their possible tasks; AVMA euthanasia guidelines; pain and distress, analgesia and anesthesia; humane endpoints; personal emotions; differences between clinic and research

$4 \quad$ Managing regulations and the animals themselves

Types of animal studies (testing, teaching, field and research: basic, applied, translational, pharmacokinetics); history of biomedical science; the 3Rs; biomedical advocates and antivivisectionist groups; welfare concerns; regulations, government agencies, regulatory bodies and accreditation

\section{Class activities and assignments}

Peruse handouts and flyers and write a reflection on one; view the AALAS and university Office of Research web sites; begin animal model worksheet; discuss the make-up of a protocol; develop the animal model and purpose for a study; sign up for Foundation for Biomedical Research eClips newsletter; skim ch. 4

Begin writing protocol; discuss ethical uses of animals; write an ethics essay on animal usage; listen to a PI presentation

Discuss animal health maintenance; learn common diseases and complete an animal health report form; add the husbandry, euthanasia, drugs and technical usage aspects to the protocol; define the specific husbandry and vet concerns pertaining to the protocol (housing, caging, bedding, diet, environment, handling and restraint, enrichment, anesthesia, etc.); research a disease or condition that the animal model can contract; examine a food and drug label; read ch. 5

Review Guide for the Care and Use of Laboratory Animals (National Academies Press, Washington, DC, 2011); review Public Health Service Assurance Statement; discuss 'whistleblower' policy; find an animal law in student's hometown; look at giveaways from biomedical advocacy groups; skim chs. 4, 6 and 16 